\title{
On Microwave Imaging with Absorbing Metasurface Enclosure
}

\author{
Ziqi Liu, Nozhan Bayat, and Puyan Mojabi \\ Department of Electrical and Computer Engineering \\ University of Manitoba, Winnipeg, Manitoba, Canada
}

\begin{abstract}
Matching fluids used in microwave imaging are often lossy to reduce the reflections from the system enclosure. To enable the use of low-loss matching fluids, we investigate the use of absorbing metasurfaces as the enclosure. This has the potential to enhance the signal-to-noise ratio of the data, thus, enhancing the achievable image accuracy. The presented example has the following limitation: as opposed to design and simulation of the physical structure of the metasurface enclosure, it uses two impedance boundary conditions on top of a metallic-backed substrate. Thus, the angular dependency of a practical absorber is not completely taken into account by this model. At the conference, we'll also consider a microwave imaging metasurface enclosure via design and simulation of a physical structure.
\end{abstract}

\section{INTRODUCTION}

In microwave imaging (MWI), the goal is to create quantitative images of the relative complex permittivity of the irradiated object of interest (OI) from the scattered field data collected outside the OI. In MWI for biomedical applications, matching fluids are often used to effectively couple irradiating microwave energy into biological tissues. The matching fluid is contained within a chamber whose walls are referred to as the MWI enclosure (or, casing). Depending on the OI, these matching fluids may be chosen to be canola oil [1], saltwater [2], glycerin-water mixtures [3], etc. Similar to indoor antenna ranges where measurements are performed in anechoic chambers, it is often desired to mitigate the reflections from the MWI enclosure. This is challenging when the utilized matching fluid has a high permittivity and/or if the enclosure is metallic. In MWI, the typical approach to minimize reflections is to make the matching fluid lossy to reduce the level of these unwanted reflected signals. For example, in [4], deionized water was considered as the matching fluid with various loss levels introduced by the addition of table salt to the water. This changed the imaginary part of the relative complex permittivity of the matching fluid from about $\operatorname{Im}\left(\epsilon_{r}\right) \approx-j 4$ to about $-j 25$ around $1 \mathrm{GHz}$. (The more negative the imaginary part, the greater the loss.) At around $-j 4$, the reflections from the metallic enclosure were too high; thus, this matching fluid was not used. On the other hand, at $-j 25$, although the reflections from the metallic casing were small, the desired signal levels were also too small; thus, this matching fluid was not used either. Finally, a trade-off imaginary value at around $-j 15$ was created and used 1

\footnotetext{
${ }^{1}$ In addition to adding loss, there exist other proposed approaches, e.g., taking into account the enclosure reflections in inversion or removing them in a preprocessing approach [5]. These alternatives are not considered herein.
}

Herein, our purpose is to investigate the use of absorbing metasurfaces [6] as the MWI enclosure. This can result in relaxing the necessity to add loss to the matching fluid; thus, enhancing the signal-to-noise ratio (SNR) of the measured data. In addition, due to the small thickness of metasurfaces, the size of the MWI enclosure does not change much, which is important for the imaging system to be small and portable. As noted in [7], several absorbing metasurface designs are based on having a thin dielectric substrate backed by a perfect electric conductor (PEC), e.g., see [8]. Although in many scenarios, the presence of the PEC-backed absorber may not be desirable, this is, in fact, advantageous for MWI since it shields the imaging system from external interferences. In addition, the narrow bandwidth of these PEC-backed metasurface absorbers are not a major issue for narrowband MWI systems. Due to the fact that in MWI we have many scattering events, it is vital for the absorbing metasurface to be operational with respect to different incidence angles. In [9], this performance stability with respect to incidence angles was achieved by making the surface impedance less dependent on incidence angles. In addition, in [10], it has been shown that a truly angle-independent zero-reflection metasurface (with a nonzero transmission) needs to be nonreciprocal and active.

\section{Simulation Results}

The frequency of operation was set to $1 \mathrm{GHz}$, and the matching fluid was considered to be deionized water with the relative complex permittivity of $75.6-j 4$. The absorbing metasurface was first simulated in the unit cell topology with periodic boundary conditions in ANSYS HFSS in the presence of the matching fluid. The unit cell consists of a PEC-backed dielectric substrate with the substrate having the same property as RO3003: the relative permittivity of 3 and the thickness of $1.52 \mathrm{~mm}$. On top of the substrate, we have used two parallel impedance sheets with the following values: $-j 11.8834$ (capacitive) and 32.6492 (resistive) ohms per square, separated by $0.2 \mathrm{~mm}$. Herein, we have modelled these two impedance sheets by HFSS impedance boundary conditions. Due to the use of this simplified model, as opposed to designing and simulating the physical structure of the impedance sheets, the resulting metasurface model is not capable to fully take into account practical absorber angular dependency. This metasurface model is then used as the enclosure of the MWI system depicted by the black square $\Gamma$ in Figure 11(a). The MWI measurement domain is denoted by $\mathcal{S}$ which consists 


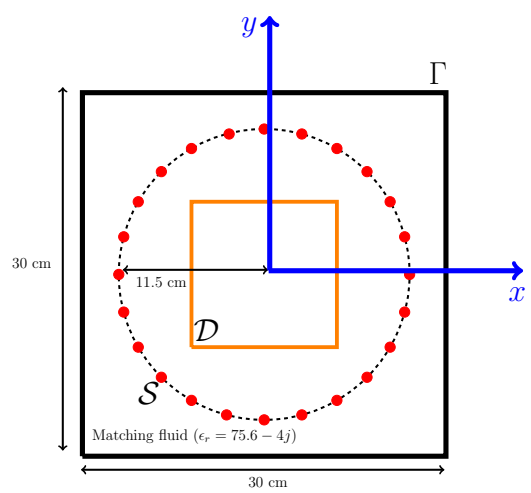

(a) Setup (not to scale)

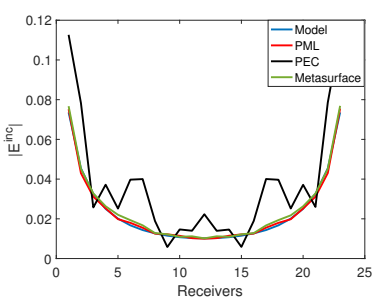

(b) $\left|E^{\text {inc }}\right|$

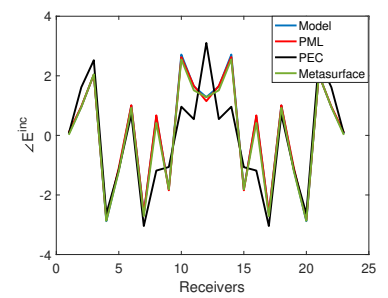

(c) $\angle E^{\text {inc }}$
Fig. 1. (a) MWI setup: $\Gamma$ is the MWI enclosure, $\mathcal{S}$ is the measurement domain, and $\mathcal{D}$ is the imaging domain. (b)-(c) The comparison of the incident field data $E^{\text {inc }}$ at 23 receivers for the transmitter at $(11.5 \mathrm{~cm}, 0,0)$.

of 24 antennas (line sources). The imaging domain denoted by $\mathcal{D}$ contains the OI which is a human forearm model consisting of a fatty layer, muscle region, and two bones. Since we consider tomographic imaging, we have placed two PEC planes on the top and bottom of the imaging system to make the system two-dimensional. Once an antenna illuminates the human forearm model, the remaining 23 antennas collect the emanating fields, resulting in $24 \times 23=552$ scattered field data points. Figure 11.b)-(c) show the incident field data for four configurations: (i) numerical model used in the imaging algorithm which is the zeroth-order Hankel function of the second kind, and the incident field data obtained under (ii) perfect matched layer (PML) enclosure, (iii) PEC enclosure, and (iv) absorbing metasurface enclosure. For the incident field data on $\mathcal{S}$, the metasurface enclosure behaves similar to the PML enclosure. This is due to (1) the presence of $-j 4$ loss in the matching fluid and (2) the fact that our simplified metasurface model is not able to fully take into account the angular dependency of a practical absorber. The scattered field data are then given to the multiplicative regularized Gauss-Newton inversion algorithm [2] to reconstruct the real and imaginary parts of the relative complex permittivity of the human forearm model. The reconstructions are shown in Figure 2 where the muscle region and the two bones are clearly visible in both real and imaginary parts of the relative complex permittivity.

\section{CONCLUSiOn AND Future WORK}

Absorbing metasurfaces might enable the use of low-loss matching fluids in MWI applications. This can then lead to an

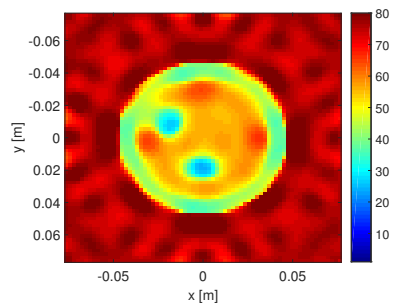

(a) $\operatorname{Re}\left(\epsilon_{r}\right)$

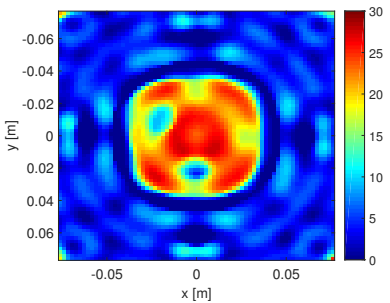

(b) $-\operatorname{Im}\left(\epsilon_{r}\right)$
Fig. 2. The reconstructed real and imaginary parts of the relative complex permittivity of the human forearm model with the metasurface enclosure.

enhanced SNR for the data. However, this approach comes at the cost of increased complexity for enclosure fabrication and a limited frequency bandwidth. For this approach to work, the metasurface needs to be operational over a wide range of incidence angles. The main limitation of our example was the use of HFSS impedance boundary conditions as opposed to simulating a physical structure, e.g., a mushroom array configuration. This approach cannot completely take into account the angular dependency of a practical absorber. Once a physical metasurface enclosure is simulated, the achievable image accuracy needs to be re-evaluated. We are currently simulating and optimizing a physical metasurface enclosure (in free space) which shows performance stability with respect to the incidence angle. This will be discussed at the conference.

\section{ACKNOWLEDGEMENT}

The financial support of NSERC Canada is acknowledged.

\section{REFERENCES}

[1] J. M. Sill and E. C. Fear, "Tissue sensing adaptive radar for breast cancer detection-experimental investigation of simple tumor models," IEEE Trans. Microw. Theory Tech., vol. 53, no. 11, 2005.

[2] M. Ostadrahimi, P. Mojabi, A. Zakaria, J. LoVetri, and L. Shafai, "Enhancement of Gauss-Newton inversion method for biological tissue imaging," IEEE Transactions on Microwave Theory and Techniques, vol. 61, no. 9, pp. 3424-3434, Sept 2013.

[3] P. M. Meaney, C. J. Fox, S. D. Geimer, and K. D. Paulsen, "Electrical characterization of glycerin:water mixtures: Implications for use as a coupling medium in microwave tomography," IEEE Trans. Microw. Theory Tech., vol. 65, no. 5, pp. 1471-1478, May 2017.

[4] C. Gilmore, A. Zakaria, J. LoVetri, and S. Pistorius, "A study of matching fluid loss in a biomedical microwave tomography system," Medical Physics, vol. 40, pp. 1-14, 2013.

[5] P. M. van den Berg and J. T. Fokkema, "Removal of undesired wavefields related to the casing of a microwave scanner," IEEE Trans. Microw. Theory Tech., vol. 51, no. 1, pp. 187-192, Jan 2003.

[6] Y. Ra'di, C. R. Simovski, and S. A. Tretyakov, "Thin perfect absorbers for electromagnetic waves: Theory, design, and realizations," Phys. Rev. Applied, vol. 3, p. 037001, Mar 2015.

[7] Y. Ra'di, V. S. Asadchy, and S. A. Tretyakov, "Total absorption of electromagnetic waves in ultimately thin layers," IEEE Transactions on Antennas and Propagation, vol. 61, no. 9, pp. 4606-4614, Sep. 2013.

[8] N. Engheta, "Thin absorbing screens using metamaterial surfaces," in IEEE APS International Symposium, vol. 2, June 2002, pp. 392-395.

[9] O. Luukkonen, F. Costa, C. R. Simovski, A. Monorchio, and S. A. Tretyakov, "A thin electromagnetic absorber for wide incidence angles and both polarizations," IEEE Transactions on Antennas and Propagation, vol. 57, no. 10, pp. 3119-3125, Oct 2009.

[10] G. Lavigne and C. Caloz, "Angle-independent nongyrotropic metasurfaces," arXiv:1903.11956, 2019. 